1 Perspectives Paper:

\title{
2 A horizon scan of priorities for coastal marine microbiome research
}

3

4 Stacey M. Trevathan-Tackett ${ }^{1 *}$, Craig Sherman ${ }^{1}$, Megan J. Huggett ${ }^{2,3}$, Alexandra H.

5 Campbell $^{4,5}$, Bonnie Laverock ${ }^{6}$, Valentina Hurtado-McCormick ${ }^{7}$, Justin Seymour ${ }^{7}$, Alana

6 Firl $^{8}$, Lauren Messer ${ }^{9}$, Tracy Ainsworth ${ }^{4,10}$, Karita L. Negandhi ${ }^{11}$, Daniele Daffonchio ${ }^{12}$,

7 Suhelen Egan ${ }^{4}$, Aschwin H. Engelen ${ }^{13}$, Marco Fusi ${ }^{12,14}$, Torsten Thomas ${ }^{4}$, Laura Vann ${ }^{8}$,

8 Alejandra Hernandez-Agreda ${ }^{10,15}$, Han Ming Gan ${ }^{1}$, Ezequiel M. Marzinelli ${ }^{4,16,17}$, Peter D.

9 Steinberg $^{4,16,17}$, Leo Hardtke ${ }^{7}$, Peter I. Macreadie ${ }^{1}$

10

$11{ }^{1}$ Deakin University, Geelong, Centre for Integrative Ecology, School of Life and

12 Environmental Sciences, Burwood and Waurn Ponds Campuses, VIC, 3130 Australia

$13{ }^{2}$ Centre for Marine Ecosystems Research and Centre for Ecosystem Management, School of

14 Science, 270 Joondalup Dr, Edith Cowan University, Joondalup 6027 WA Australia

$15{ }^{3}$ School of Environmental and Life Sciences, The University of Newcastle, 10 Chittaway Rd,

16 Ourimbah 2258 NSW Australia

$17{ }^{4}$ Centre for Marine Bio-Innovation, School of Biological, Earth and Environmental Sciences,

18 University of New South Wales, Sydney NSW 2052, Australia

$19{ }^{5}$ Faculty of Science, Health, Education \& Engineering, University of the Sunshine Coast,

20 QLD 4556, Australia

$21{ }^{6}$ School of Science, Auckland University of Technology, Private Bag 92006, Auckland, New

22 Zealand

$23{ }^{7}$ Climate Change Cluster, University of Technology Sydney, 15 Broadway, Ultimo, NSW

24 2007, Australia

$25{ }^{8}$ Genome Center, University of California, Davis, California 95616 
$26{ }^{9}$ Australian Centre for Ecogenomics, University of Queensland, St Lucia, Brisbane, QLD, 27 4072, Australia

$28{ }^{10}$ Australian Research Council Centre of Excellence for Coral Reef Studies, James Cook

29 University, Townsville, QLD, 4811, Australia

$30{ }^{11}$ Department of earth and planetary sciences, Macquarie University, North Ryde, NSW

312109 , Australia

$32{ }^{12}$ King Abdullah University of Science and Technology (KAUST), Red Sea Research Center

33 (RSRC), Thuwal 23955-6900, Saudi Arabia

$34{ }^{13}$ Centre for Marine Sciences (CCMAR), Universidade do Algarve, Campus de Gambelas,

35 8005-139 Faro, Portugal

$36{ }^{14}$ School of Applied Sciences, Edinburgh Napier University, Edinburgh, UK

$37{ }^{15}$ The College of Public Health, Medical and Veterinary Sciences, James Cook University,

38 Townsville, QLD, 4811, Australia

$39{ }^{16}$ Sydney Institute of Marine Science, 19 Chowder Bay Rd, Mosman, NSW 2088, Australia

$40 \quad{ }^{17}$ Singapore Centre for Environmental Life Sciences Engineering, Nanyang Technological

41 University, Singapore 637551

42

$43 *$ Corresponding Author. E-mail: s.trevathantackett@deakin.edu.au 


\section{Abstract}

46 Research into the microbiomes of natural environments is changing the way ecologists and

47 evolutionary biologists view the importance of microbes in ecosystem function. This is

48 particularly relevant in ocean environments, where microbes constitute the majority of

49 biomass and control most of the major biogeochemical cycles, including those that regulate

50 the Earth's climate. Coastal marine environments provide goods and services that are

51 imperative to human survival and well-being (e.g. fisheries, water purification), and emerging

52 evidence indicates that these ecosystem services often depend on complex relationships

53 between communities of microorganisms (the 'microbiome') and their hosts or environment

54 - termed the 'holobiont'. Understanding of coastal ecosystem function must therefore be

55 framed under the holobiont concept, whereby macroorganisms and their associated

56 microbiomes are considered as a synergistic ecological unit. Here we evaluated the current

57 state of knowledge on coastal marine microbiome research and identified key questions

58 within this growing research area. Although the list of questions is broad and ambitious,

59 progress in the field is increasing exponentially, and the emergence of large, international

60 collaborative networks and well-executed manipulative experiments are rapidly advancing

61 the field of coastal marine microbiome research.

64 Keywords: bioremediation, core microbiome, dysbiosis, functional diversity, environmental 65 stress, evolution, holobiont, microbial ecology 


\section{Background}

71 Coastal marine ecosystems provide a range of ecologically and economically important

72 ecosystem services, including habitat provisions, nutrient cycling, coastal protection and

73 fisheries enhancement ${ }^{1}$. The health and services of these ecosystems are inherently linked to

74 the microorganisms residing in these ecosystems (e.g. pollution remediation, disease and drug

75 discovery ${ }^{2-4}$ ). As we increase our understanding of the importance of coastal marine

76 microorganisms and their genetic makeup (i.e. the microbiome, see Box 1), the number of

77 research articles describing the distribution, structure, and function of microbiomes

78 associated with coastal marine ecosystems has flourished (Supplementary Figs. 1 and 2). The

79 ecosystem services are largely attributed to the habitat-forming organisms, such as corals,

80 sponges, macroalgae, seagrasses, mangroves and saltmarshes, which form the foundation of

81 these ecosystems. Furthermore, due to the reliance of coastal marine ecosystem health on

82 these habitat-forming organisms, the field has realized the importance of understanding the

83 macroorganisms and their microbiomes as a synergistic ecological unit (i.e. holobiont, see

84 Box 1). As a result, there has been a relative surge in host-associated microbiome research in

85 recent years (Supplementary Fig. 1) aimed at identifying how microbiomes influence host

86 phenotype, physiology, and development ${ }^{5-7}$. Although our understanding of several

87 fundamental concepts in coastal marine microbial ecology has increased ${ }^{7,8}$, coastal

88 microbiome research -- particularly in the context of holobionts -- is still in its infancy,

89 especially relative to other microbiome fields, such as the human microbiome. A large

90 number of open questions currently limits our capacity to assess how microbial processes

91 influence the ecology of these environments, both under contemporary conditions and under

92 future environmental change. Therefore, there is a clear need to prioritize and define key

93 questions for future research that will allow for better assessments of how microbial

94 processes truly influence the ecology and health of coastal marine environments. 
96 [suggested Box 1 placement]

97

\section{Evaluating the state of the science}

99 To evaluate the current state of coastal marine microbiome research, we surveyed the current

100 literature, then 'horizon scanned' with experts in the field to identify major research gaps, in

101 order to determine where future challenges lie and ultimately progress this field of research

102 (see Box 2 for description of the approach and limitations). For the literature search, we

103 focused on six key holobionts that form the foundation of these coastal ecosystems - corals,

104 sponges, macroalgae, seagrasses, mangroves and saltmarshes. We also considered the

105 microbiomes of sediments and the water column within coastal marine ecosystems. The key

106 findings from the literature survey include identification of areas of progress, as well as

107 holobiont systems that need more attention (Supplementary Figs. 1 and 2). For example,

108 research on seawater- and sediment-associated microbiomes has dominated coastal marine

109 microbiome literature to-date (consistently $\geq 50 \%$ of the total number of studies), while host-

110 associated microbiome research is steadily increasing and has generally focused on coral and

111 sponge holobionts (Supplementary Fig. 1). In the last five years, however, the diversity and

112 quantity of microbiome and holobiont research has incrementally increased with the inclusion

113 of macrophyte-associated microbiome studies, although mangrove- and saltmarsh-associated

114 microbiome research is still nascent (Supplementary Fig. 1). Additionally, the methodologies

115 used to describe coastal marine microbiomes has diversified over time from predominantly

116 microscopy, cell counts, and community fingerprinting techniques, to sequencing-dominated

117 technologies (Supplementary Fig. 1). The literature survey also identified geographic

118 hotspots and gaps in microbiome studies (Supplementary Fig. 2). The coastlines of Australia,

119 Europe, the northern Mediterranean Sea, the Red Sea and US are relatively well-sampled in 
120 multiple ecosystem types, while there are clear regional gaps for host-associated microbiome 121 studies along the South American, African and northern Asian coastlines. Some of the well122 studied regions are dominated by studies on specific host-associated microbiomes

123 (Supplementary Fig. 2). For instance, seagrasses have been heavily studied in the temperate

124 US, while the biodiversity hotspots in the Indo-Pacific have been dominated by studies on

125 coral- and sponge-associated microbiomes (Supplementary Fig. 2).

127 The horizon scan resulted in 108 questions key to progressing coastal marine microbiome 128 research. Nearly half of the questions $(\sim 50)$ directly or indirectly concerned host-associated 129 microbiomes, with the remaining covering a range of fundamental microbiome ecology or 130 methodological topics independent of a specific ecosystem, host or substrate. In assessing the 131 literature and identifying priority research questions via the horizon scanning exercise (see 132 Box 2 for the methodology used), we outline seven microbiome research themes relevant to 133 deciphering the role of microbiomes within coastal marine ecosystems. The themes begin 134 with microbiome questions, followed by host-microbiome themes, and lastly questions 135 concerning microbiomes and holobionts in the environment (Box Diagram 1). While some of 136 the themes are holobiont-centric, we do not focus on one particular holobiont system. Rather 137 the themes represent general concepts that can be applied to multiple substrate- or host138 associated, or free-living microbiome systems. Therefore, we have provided a diversity of 139 references to support the presented themes throughout, with the aim to create a comprehensive vision that may unify the strategy of research on coastal marine microbiomes. 
142 [suggested Box 2 placement]

143

144 Key research themes in coastal marine microbiome research

\section{Microbiome}

Theme 1: How can community structure be matched to microbiome function?

149 In coastal marine ecosystems, enormous microbial diversity has been revealed via, for

150 instance, phylogenetic analyses of the $16 \mathrm{~S}$ rRNA gene (e.g. ${ }^{8-10}$ ). However, it is important to

151 define the function of a microbiome in order to understand how it is likely to influence its

152 host and the ecosystem ${ }^{11}$. Currently, the best way to directly determine the function of the

153 entire microbiome is via metagenomic and metatranscriptomic sequencing ${ }^{12-16}$. The recent

154 availability of many genome reconstruction or binning approaches ${ }^{17}$ offers a greater capacity

155 to obtain near-whole genomes out of metagenomes, allowing a better understanding of the

156 function of the microbiome members. However, our ability to successfully annotate

157 functional genes within metagenomic and metatranscriptomic datasets remains outstripped by

158 the availability of sequencing data itself. For example, extensive sequencing of the global

159 ocean microbiome found that $40 \%$ of core orthologous genes were of unknown function ${ }^{18}$.

160

161 Another approach to link diversity with function is to identify the 'core microbiome', or the

162 persistent and functionally essential members of host-associated microbiomes, possibly a key

163 determinant of host well-being and therefore overall ecosystem functioning and health (Fig.

$1641)^{8,19}$. For example, conserved bacterial taxonomic groups, which constitute the coral core

165 microbiome, play a critical role in the success of the coral-zooxanthellae symbiosis ${ }^{19}$. Other 
organisms, such as the green seaweed Ulva mutabilis, require a core set of functions from their microbiome, rather than the presence of specific taxa $\mathrm{a}^{20}$.

Connecting diversity with function drives central ecological questions such as: (1) How does microbial community diversity influence functional aspects (e.g. resilience) of the host, microbiome and environment; and (2) How can we define the function of a coastal microbiome? Yet despite substantial effort in recovering metagenomes and metatranscriptomes from dominant marine hosts, there remain significant challenges in demonstrating causation between shifts in the microbiome and shifts in host health due to reduced capabilities to manipulate microbiomes in the field ${ }^{18}$ (e.g. manipulative field experiments, see Box 3). We recognize this as a particular challenge for microbial ecologists. Therefore, we identify several questions that we hope will move the field forward and lead into innovative approaches that determine the functional roles of coastal marine microbiomes, and thereby resolve the relationship between microbiome diversity and their functions:

- How can novel techniques, e.g. single cell raman spectroscopy ${ }^{21}$, be applied to complex microbiomes and holobionts to interrogate microbial functions in situ?

- How can intensive efforts for isolating coastal and host-associated microbes (i.e. 'culturomics ${ }^{, 22}$ ) open the door for tracking the function of candidate genes and investigating homology, predictability and certainty of curated gene function?

- How does identifying the core microbiome (taxa or set of functions, e.g. depicted in Fig. 1b-c, respectively) offer valuable insights towards advancing in-depth identification and experimental manipulation?

\section{[suggested placement for Figure 1]}



change?

192

Host-associated microbiomes are highly dynamic communities that change at both small (i.e. ecological and physiological) and large (i.e. evolutionary and geographical) timescales.

195 Substantial variability on very small spatial scales (i.e. within host) can be driven by host 196 provisions, such as nutrient and oxygen availability ${ }^{10,23}$, as well as by trophic- and quorum 197 sensing-related interactions among members of the microbiomes within a physical niche (e.g. $\left.{ }^{24,25}\right)$. Hosts also differ in microbiome community structure depending upon host distribution in a population (e.g. center vs. edge of a seagrass meadow ${ }^{26}$ ), and microbiomes on host species can also vary across large environmental gradients ${ }^{27}$. However, for some holobionts such as seaweeds, geographical variability in surface-associated microbiomes is relatively low even at continental scales, relative to other factors such as host health condition ${ }^{9}$. Shortterm temporal variability can also be surprisingly consistent, with predictable successional patterns over periods of days to weeks occurring in the epiphytic bacterial communities of macroalgae ${ }^{28}, \operatorname{corals}^{29}$ and sponges ${ }^{30}$. However, evidence for the scales at which coastal marine microbiomes shift in time and space, and the apparent drivers behind these shifts, is

207 often conflicting. Studies showing host-specificity and stability in the microbiome over time 208 and location $^{10,28,31,32}$ contradict studies that suggest that microbial communities are highly 209 dependent on the host physiological or environmental conditions ${ }^{9,33,34}$. These conflicting 210 patterns prevent us from making generalizations about the stability or variability of coastal

211 host-associated microbial communities (e.g. Theme 5).

213 At evolutionary time scales, there is little doubt that hosts and their associated microbiomes

214 influence each others' evolution, and indeed at very large time scales, these interactions are 
215 the basis for fundamental macroevolutionary events ${ }^{35}$. For individual marine systems,

216 however, the details of how hosts and their microbial associates affect each other on shorter

217 evolutionary time scales is limited, and whether or not these effects are broadly reciprocal

218 (i.e. coevolutionary). To date, there is limited evidence among benthic marine hosts for

219 coevolution with their microbiome $\mathrm{e}^{34,36}$, both because of the multiple interplaying factors that

220 ultimately influence evolutive patterns, and because of the challenges in demonstrating

221 formal coevolutionary relationships ${ }^{37}$. In some instances broad taxa of marine hosts and their

222 microbiota appear to reciprocally evolve in response to one another ${ }^{38}$, but in others selective

223 effects appear to be limited to the host and individual members of its microbiome rather than

224 the entire microbial community ${ }^{39}$. Further complexities in teasing out the evolution of

225 members in a holobiont include both internal microenvironments of the host that act like

226 discrete coevolving ecosystems ${ }^{40}$, as well as the disparate evolutionary timescales that

227 influence the host and the diversity of the microbiome members ${ }^{41}$. Evolutionary patterns

228 within holobiont spatial niches/compartments have been shown for Scleractinian corals and

229 their microbiomes, whereby the ecological relatedness of host-associated microbial

230 communities parallels the phylogeny of related host species, and therefore evolutionary

231 changes in the host associate with ecological changes in the microbiota ${ }^{42}$. This

232 coevolutionary pattern, or phylosymbiosis was strongest in the coral skeleton compared to the

233 coral tissue and mucus ${ }^{43}$. Although many coral-associated bacteria were host-specific, only a

234 select minority of coral-associated bacterial families showed co-phylogenetic signals

235 consistent with long-term host-microbe co-diversification ${ }^{43}$.

236

237 Here, we outline key questions to progress our understanding of the scales at which

238 microbiomes shift: 
- What are the implications of disparate evolutionary timescales between the host and its microbiota?

- How does the resolution at which we study microbiomes influence how we interpret differences in their composition and function at spatial and temporal scales?

- Do the holobiont members differentiate between beneficial and detrimental

248 To improve our understanding of the temporal and spatial dynamics of host-associated 249 microbiomes, a structured approach to characterize spatial and temporal variation at multiple 250 scales for both taxonomic and functional characteristics is needed. Future research should 251 focus not only on descriptive studies, but also on perturbation experiments to assess resilience 252 and stability under the context of variable systems ${ }^{44-46}$. Investigating microbiome evolution is 253 inherently challenging, therefore clearly defining the boundaries of the question (e.g. phylogenetic vs functional level or the whole microbiome vs individual members), as well as identifying the limitations of what can be tested is necessary. Additional reflection on (co)evolution in systems other than coastal marine ecosystems may provide insight that could 257 progress these questions. Examples include the formation of niches by symbiotic microbiota ${ }^{40}$ 258 and the broader literature on geographic aspects of coevolution (e.g. ${ }^{47}$ ).

Microbiome and Host 
264 Several studies have established that most benthic organisms, including seagrasses ${ }^{10}, \operatorname{corals}^{31}$

265 and macroalgae ${ }^{48}$, carry microbiomes that are distinct from the surrounding sediment or

266 seawater. Yet, the timing and underlying mechanisms of microbiome acquisition (either host-

267 directed selection, or microbe-direct colonization) remain largely unresolved. Chemical

268 signaling, specifically secondary metabolites produced by host species independently or in

269 response to environmental or microbial cues, or signaling from microbial taxa that have

270 already colonized the host, have been suggested to be important factors in both host defense

271 against pathogenic microbes and microbiome colonization. For example, the pathogen Vibrio

272 coralliilyticus has been shown to be attracted to corals that increase their production of the

273 sulfur compound dimethylsulfoniopropionate (DMSP) under heat stress ${ }^{49}$. Conversely, in

274 seaweeds such as Lobophora variegata, secondary metabolite production acts as a defense

275 strategy by preventing colonization of pathogenic microbes, such as saprophytic marine

276 fungi ${ }^{50}$.

277

278 In addition to host-microbe interactions, some studies have suggested a role for microbe-

279 microbe interactions in determining microbiome composition, including lottery models ${ }^{51}$ and

280 symbiotic modes of interaction ${ }^{31}$. It has also been shown that microbiome composition is

281 affected by host condition (e.g. seaweed ${ }^{9}$; $\operatorname{corals}^{49}$ ), as well as environmental conditions (see

282 Theme 5). Although there are few global census studies of the microbiome of particular

283 marine species, recent studies in seagrasses ${ }^{10,33}$ suggest that microbial functions and

284 microbiome composition are also affected by geographic location, indicating an influential

285 role of the environment in shaping microbiome composition. Taken together, we hypothesize

286 that the active role of the host in determining microbiome composition lies along a

287 continuum, ranging from being determined by host condition to being determined by

288 environmental factors, which no doubt affect host condition. Where the system lies within the 
continuum is largely determined by host species. Additional studies in coastal marine ecosystems are needed to elucidate further:

- What are the differing selection strategies between host species that determines whether the microbiome is shaped more by the environment or by the host?

- What are the chemical pathways or specific processes by which a host attracts specific microbes, e.g. as observed in the model organism Arabidopsis microbiome ${ }^{52}$ ?

- Does the host species dynamically change its selection strategies as a function of microbial colonization, or changing environmental conditions?

To address these questions, controlled experiments in mesocosm systems are needed for the use of model organisms and standardized initial conditions. Tuneable manipulation of environmental parameters, addition of other microbial species, comparing a variety of host genotypes, and characterizing host exudate composition, could elucidate mechanistic interactions between host and microbiome, and discern the conditions under which a mechanism can be expected to occur. Some studies have made use of mesocosm systems ${ }^{44,53,54}$, and we expect even further advances from the use of controlled systems.

Theme 4: To what extent is the resilience and health of the holobiont determined by the structure and function of its microbiota?

The importance of microbes to the health of plants and animals is now well accepted. The microbial components of the holobiont can aid in digestion, provide essential vitamins and nutrients, protect from invading pathogenic organisms and stimulate developmental processes $^{7,55,56}$. Therefore, any disturbance to the host microbiome are likely to result in a 
313 breakdown of holobiont function (or dysbiosis), which can manifest itself as disease.

314 Analogous disease concepts have been proposed for chronic conditions in humans, including

315 common periodontal and gastrointestinal disorders, which are thought to result from a

316 disturbance to the natural microbiota rather than infection by a singular pathogen ${ }^{57,58}$. While

317 less well understood for marine holobionts, microbial dysbiosis may also play a role in

318 diseases, for example, the bleaching diseases of invertebrates and seaweeds (e.g. see recent

319 reviews $^{59,60}$ ). However, with some exceptions ${ }^{61}$, the majority of these observations are based

320 on correlative data, and the extent to which disease is a direct result of microbial dysbiosis

321 remains an important research question. To fully appreciate the role of microbial dysbiosis

322 we need to understand the core components of a healthy microbiome and identify those

323 beneficial consortia that offer holobiont resilience. Importantly, given the capacity of

324 microbes to rapidly respond, adapt and evolve to environmental conditions, the host

325 microbiome is also likely to be instrumental in assisting the adaptation of higher organisms to

326 future climate conditions or other anthropogenic stressors ${ }^{62}$.

327

328 Structure and function of the microbiota within a holobiont can have important links to

329 broader scale holobiont health and resilience. These connections are likely to aid in

330 identifying core microbiome members and their corresponding functions essential for

331 holobiont health (i.e. Theme 1). As we move to a changing climate, several key questions

332 remain:

333 - How do the interactions among microbiomes, within or across different niches of the

$334 \quad$ same host affect host, resilience and homeostasis?

335 - What are the criteria to designate specific taxa as beneficial core microbiome

336 members or sentinels of dysbiosis in marine organisms? 
- To what degree are members of the transient microbiome a source of functional redundancy and thus providing resilience during environmental change?

Looking to the future, having sound knowledge and access to culturable, beneficial members of the core microbiota will have applied uses; for example, as biomarkers for the early detection of host stress or for the development of probiotic consortia that can be used to support aquaculture and marine restoration programs (Theme 6). However, taxa not considered part of the core microbiome under current conditions may become more important (core) under future conditions.

\section{Microbiome, Holobiont \& the Environment}

Theme 5: What is the role of the tripartite interaction, host-microbiome-environment, on holobiont resilience?

Holobionts living in the dynamic ocean-land interface of coastal ecosystems ${ }^{63}$ can be exposed to substantial diel changes in temperature, salinity, tidal levels, light, oxygen, and nutrients ${ }^{64}$. Their resilience and adaptation is at least partially influenced by the microbiomes that modulate the environmental conditions to which they are exposed ${ }^{65}$. The environment can act as a source for holobiont microbiota that in turn are shaped by strong selective forces driven by the host biology and behaviours. For example, fiddler crabs carapace and gut ${ }^{66}$ are colonized by different pools of microbial colonists that are taken up from the environment, 
361 but the burrowing and filter feeding behaviors of the crabs finely select such colonists from

362 the sediment after strongly reconditioning its geochemical properties ${ }^{67}$.

363

364 The effects of either short- or long-term environmental changes on host-microbiome

365 interactions are inherently complex and thus difficult to predict ${ }^{68}$. The intrinsic environmental

366 variability, for instance linked to seasonal changes ${ }^{69}$, perturbation events ${ }^{70}$, or a combination

367 of these ${ }^{71}$, strongly influence microbiome diversity and functionality. Environmental stressors

368 that can interact in opposing, additive or synergistic ways to influence hosts, microbiomes

369 and their interactions, can lead to positive, negative or neutral impacts on them ${ }^{72}$.

370

371 Using as an example thermal stress, frequently investigated in coastal marine microbiome

372 research, we should consider that all organisms, whether microbial or macroscopic, have

373 optimal thermal tolerance thresholds ${ }^{73}$. Thermal stress has been correlated with functional

374 and/or structural shifts in microbiomes of corals ${ }^{74}$, pponges $^{75}$ and oysters ${ }^{76}$, among others.

375 Higher temperatures can induce virulence in otherwise commensal microbes ${ }^{77}$, and/or

376 decrease the host chemical defences, with continued stress leading to the break-down of

377 symbioses, the introduction of new microbes (e.g. opportunistic pathogens) and, eventually,

378 deterioration of the host $\mathrm{t}^{61,75}$.

379

380 The ecological interactions within and among holobionts can also be indirect, for instance,

381 microbiome recruitment by one host that may be affected by the exudates of another nearby

382 host $^{23,78}$. Host proximity may affect microbiome compositions, such as for algal turfs on the

383 surface of Porites coral that were associated with increased alpha diversity of coral surface

384 microbes, particularly of pathogenic bacterial taxa ${ }^{79}$. Host coexistence may also provide a

385 more suitable habitat, e.g. seagrasses in anoxic sediments are favored by the aerobic sulfide- 
386

387

388

389

390

391

392

393

394

395

396

397

398

399

400

401

402

403

404

405

406

407

408

409

808

oxidizing bacterial symbionts associated with benthic burrowing bivalves, which detoxify the anoxic sediment ${ }^{80}$.

Such tripartite interactions are highly complex and challenging to investigate in 'real-world' scenarios. We envisage the following research questions as priorities for the future research on coastal marine microbiomes:

- How do environmental changes and stressors shape the functional redundancy of coastal microbiomes?

- What are the environmental factors that determine and select microbiome members as beneficial or harmful for a host?

- What are the chemical signals and how do they modulate the ecological interactions of microbiomes within and between holobionts and microbiomes?

Investigations using real-world scenarios like those on combined multi-stressors, such as heat, $\mathrm{pH}$ (ocean acidification), and oxygen availability, are still rare ${ }^{68,81}$. However, in order to address the above question, such approaches are essential to build more ecologically reliable models on how host-microbiome interactions respond and adapt to changes ${ }^{82}$. Additionally, the holobiont approach sets a research framework, to comprehensively explore the adaptive and evolutionary patterns of organismal resilience and ecological function, in response to the critical challenges imposed by multiple combined environmental changes ${ }^{83,84}$. 
410 Theme 6: How can we 'manage' microbiomes in the coastal environment and in association

411 with hosts?

412

413 Management or manipulation of microbial functions and communities are well-established

414 techniques in bioremediation of terrestrial and aquatic ecosystems - for instance, those

415 impacted by hydrocarbons and toxins contamination ${ }^{85}$. Principal approaches involve either

416 biostimulation (the process of 'activating' indigenous microbes via, for example, nutrient

417 amendments) or bioaugmentation (the process of inoculating the ecosystem with non-

418 indigenous microbes that have desired metabolic properties ${ }^{85}$ ), which have both been applied

419 at ecosystem scales ${ }^{86,87}$. While these approaches have been less well-tested for marine

420 systems, biostimulation strategies have been applied to deal with oil spills in ocean waters

421 (e.g. Exxon Valdes or Deep Horizon disasters), primarily by supplying growth limiting

422 nutrients, such as phosphate, to the site of contamination ${ }^{86}$. Biostimulation and

423 bioaugmentation have also been used to accelerate degradation of polycyclic aromatic

424 hydrocarbons in marine coastal sediments ${ }^{88}$.

426 Host-associated microbial communities can also in principle be managed, as exemplified over

427 the last few years by the development of sophisticated pre- and probiotic strategies for

428 disease prevention and health improvement of humans, plants and some aquaculture

429 species $^{89-91}$. The advances in these hosts are facilitated in an increasingly detailed

430 understanding of microbial diversity and functional processes, but such information is sparse

431 for most natural marine hosts, thus preventing rational designs of pre- and probiotic

432 strategies. ${ }^{62,92}$ Taken together, we envisage the following questions to be areas of increasing

433 research: 
- What are the key conditions to establish microbial-driven bioremediation processes in the coastal environment?

- How does microbial manipulation in the early lifecycle stages of a host influence the performance and health of more mature host stages?

- What is the role of microbial communities in facilitating the restoration of key hosts

442 With the increasing urbanization of our coastlines and the increasing need to use polluted 443 sites for recreational, private or commercial purposes, microbial-driven bioremediation will

444 be one of the key tools to tackle this issue. Engagement and involvement of the local and

445 regional stakeholders in the early stages of research will be essential for successful

446 implementation. Additionally, as there is an increasing interest in developing probiotics or

447 improving microbial symbiont function of important habitat-forming holobionts, global

448 networks or initiatives, such as the Beneficial Microorganisms for Marine Organisms

449 (BMMO), are powerful tools to progress this work, as has been shown for corals ${ }^{62,92}$. 450

Theme 7: To what extent are coastal marine holobionts and their interactions with the environment relevant to human health and well-being?

454 Coastal environments, including their associated biota, are the principal interface for human exposure to marine microbiomes and these interactions can sometimes have detrimental impacts on human health (Fig. 2). Human pathogens present within marine microbiomes

457 include both indigenous marine microbes and enteric microbes that are exogenously

458 introduced to coastal habitats via sewage and urban storm-water ${ }^{93}$ (Fig. 2). The microbiomes 459 of benthic marine flora and fauna often display a high representation of marine pathogens - in 
460 particular members of the Vibrio genus ${ }^{94}$. Several species within this genus are highly

461 virulent and dangerous human pathogens, and in the USA alone are cumulatively responsible

462 for health costs exceeding \$250 million $\mathrm{yr}^{-1}{ }^{19}$. In addition to native marine microbes, enteric

463 pathogens that become, at least transiently, incorporated into marine microbiomes following

464 exposure to coastal pollution also pose a significant health risk. Indeed, due to (i) the

465 preference for coastal and estuarine habitats among many native marine pathogens ${ }^{94}$ and (ii)

466 the regular exposure of coastal flora and fauna to human waste streams, the microbiomes of

467 coastal organisms represent potentially important hotspots and reservoirs of human

468 pathogens ${ }^{93}$ (Fig. 2). On the other hand, there is recent evidence that some marine macro-

469 organisms, specifically seagrasses, may act as effective natural filtration systems that remove

470 human pathogens from coastal ecosystems, potentially through the production of biocides by

471 the plant or its microbiome ${ }^{96}$ (Fig. 2).

472

473 As the global human population rapidly increases its dependence and impact upon coastal

474 environments ${ }^{97}$, it is imperative that we develop an understanding of the potential human

475 health consequences of increasing contact with marine microbiomes. This is particularly true,

476 given that there is emerging evidence that climate change and the anthropogenic degradation

477 of coastal habitats are enhancing the occurrence and virulence of dangerous human pathogens

478 within these ecosystems ${ }^{98}$ (Fig. 2). Within this specific context, we identify several key

479 questions that remain unanswered, including:

480

481 - Are potential human pathogens persistent or ephemeral members of the microbiomes

$482 \quad$ of coastal organisms?

483 - To what extent are environmental change and degradation enhancing the occurrence,

484 persistence and virulence of human pathogens within coastal microbiomes? 
- To what degree do enteric pathogens introduced to coastal microbiomes via human waste streams influence the health of the benthic coastal macro-organisms?

487

488 New analytical approaches for interpreting microbiome data provide several opportunities to 489 answer these questions. For instance the detection of novel "indicator" organisms ${ }^{99}$ or 490 genes ${ }^{100}$ within microbiome data-sets delivers potentially powerful capacity to detect 491 environmental perturbations and human contamination within coastal waters that goes far 492 beyond standard indicators of human contamination (i.e. Faecal Indicator Bacteria [FIB]), 493 which are often limited in sensitivity and explanatory power ${ }^{101}$. The analysis of coastal 494 microbiome data also provides a facility to detect novel or emerging pathogens that are missed by standard FIB monitoring approaches ${ }^{102,103}$. Finally, the application of analytical approaches such as SourceTracker ${ }^{104}$ and random forest analyses ${ }^{105}$ allow for microbiome

497 data to be directly used as a sensitive new tool for tracking sources of contamination or 498 signals of environmental change.

[suggested placement for Figure 2]

$501 \quad$ [suggested placement for Box 3]

502

\section{Synthesis and Outlook}

504 Coastal marine microbiome research represents a direct pathway to understanding how 505 microbes affect - both positively and deleteriously - the coastal ecosystems on which human 506 populations so heavily rely. The themes and questions presented here, summarized in a 507 conceptual framework (Fig. 3), include resolving the spatial, temporal, and evolutionary 508 scales at which the holobionts and microbiomes function, resolving how holobionts change in 509 response to environmental stimuli and each other, and determining the scope for how 
510 microbiomes can be managed. Summarizing the future of coastal microbiome research

511 through the horizon scan and literature survey has identified two overarching concepts

512 common across the themes that reflect the current state of the science, as well as how we

513 envision the science will progress: microbiome function and utilizing manipulative

514 approaches.

515

516 Defining microbiomes, either functionally or within the framework of a core microbiome,

517 was a fundamental concept shared by all the themes. As outlined in Theme 1 and the

518 literature survey, the field has made large strides in how we define microbiomes via

519 taxonomic descriptions from amplicon sequencing. For some holobionts and ecosystems like

520 mangrove and saltmarshes, gathering basic information on what microbiota are present and

521 how they may be functioning is still lacking and would benefit from global-scale initiatives,

522 such as recent efforts for seagrasses and sponges ${ }^{10,106}$. Conversely, the microbiome and

523 holobiont systems that already have solid taxonomic foundations are looking to investigate

524 how the microbiota function, alone and together with their hosts, in coastal marine

525 ecosystems in order to answer the pressing ecological questions presented.

526

527 Such investigations, as shown throughout the themes, are inherently complex, whereby the

528 questions and concepts presented in one theme relied on the understanding of another theme.

529 For example, teasing apart the relationship between microbiome and host health and

530 resilience (Theme 4) depends on the temporal scale (Theme 2) and environmental conditions

531 (Theme 5) that influence the interactions, but each of these themes in themselves also

532 influence how microbiota are selected and form holobionts (Theme 3). The ever-changing

533 nature of the ecological processes that influence the microbiomes and holobionts in the

534 natural environment necessitates manipulative experimental approaches in order to tease 
apart the questions presented. In some cases, such as the evolutionary, multi-stressor or management questions, highly controlled experiments are the best options currently available

537 to progress the respective themes. Here, the use of model organisms may provide insight, for 538 example, on selection mechanisms between host and microbe (Theme 3), and microbiome-

539 driven restoration (Theme 6). The large national and international collaborations or

540 consortium efforts that have produced the descriptive data on environmental microbiomes to-

541 date, may be equally useful in progressing hypothesis-driven questions through concerted

542 manipulative experimental approaches ${ }^{107}$, e.g. temperature effects on holobiont resilience at

543 the biogeographic limits of the host (Themes, 5, 4 and 2, respectively) or how holobionts can

544 act as sources or sinks of pathogenic microbiota under various point source or diffuse

545 pollution scenarios (Theme 7). In summary, although the list of research themes we present

546 here is broad and ambitious, the ongoing collaborative networks along with well-executed

547 hypothesis-driven manipulative experiments are significantly progressing the definition and

548 functional relationship between the core microbiome and host, illuminating global

549 microbiome biogeography, and identifying key regional- and global-scale environmental

550 influences on coastal marine microbiomes and holobionts.

551

$552 \quad$ [suggested placement for Figure 3]

553

554 Data availability: The original questions for the horizon scan are available in the 555 supplementary materials.

556

557 Code availability: The code used the extract literature from databases is available in the 558 supplementary materials. 
5611 Liquete, C. et al. Current status and future prospects for the assessment of marine and coastal ecosystem services: a systematic review. PloS One 8, e67737 (2013).

2 Harvell, C. et al. Emerging marine diseases--climate links and anthropogenic factors. Science 285, 1505-1510 (1999).

3 Penesyan, A., Kjelleberg, S. \& Egan, S. Development of novel drugs from marine surface associated microorganisms. Marine Drugs 8, 438-459 (2010).

4 Dash, H. R., Mangwani, N., Chakraborty, J., Kumari, S. \& Das, S. Marine bacteria: potential candidates for enhanced bioremediation. Applied Microbiology and Biotechnology 97, 561-571 (2013).

5 Hentschel, U., Piel, J., Degnan, S. M. \& Taylor, M. W. Genomic insights into the marine sponge microbiome. Nature Reviews Microbiology 10, 641 (2012).

6 Seymour, J., Laverock, B., Nielsen, D., Trevathan-Tackett, S. \& Macreadie, P. in Seagrasses of Australia 343-392 (Springer, 2018).

7 Bourne, D. G., Morrow, K. M. \& Webster, N. S. Insights into the coral microbiome: underpinning the health and resilience of reef ecosystems. Annual review of microbiology 70, 317-340 (2016).

8 Hernandez-Agreda, A., Gates, R. D. \& Ainsworth, T. D. Defining the core microbiome in corals' microbial soup. Trends in Microbiology 25, 125-140 (2016).

9 Marzinelli, E. M. et al. Continental-scale variation in seaweed host-associated bacterial communities is a function of host condition, not geography. Environmental microbiology 17, 4078-4088 (2015).

10 Fahimipour, A. K. et al. Global-scale structure of the eelgrass microbiome. Applied and environmental microbiology 83, e03391-03316 (2017).

11 Tremaroli, V. \& Bäckhed, F. Functional interactions between the gut microbiota and host metabolism. Nature 489, 242-249 (2012).

12 Fernandes, N., Steinberg, P., Rusch, D., Kjelleberg, S. \& Thomas, T. Community structure and functional gene profile of bacteria on healthy and diseased thalli of the red seaweed Delisea pulchra. PloS one 7, e50854 (2012).

13 Parks, D. H. et al. Recovery of nearly 8,000 metagenome-assembled genomes substantially expands the tree of life. Nature Microbiology 2, 1533-1542 (2017).

14 Tout, J. et al. Variability in microbial community composition and function between different niches within a coral reef. Microbial ecology 67, 540-552 (2014).

15 Moitinho-Silva, L. et al. Integrated metabolism in sponge-microbe symbiosis revealed by genome-centered metatranscriptomics. The ISME Journal 11, 16511666 (2017).

16 Thompson, L. R. et al. A communal catalogue reveals Earth's multiscale microbial diversity. Nature 551, 457, doi:10.1038/nature24621

https://www.nature.com/articles/nature24621\#supplementary-information (2017).

17 Sieber, C. M. et al. Recovery of genomes from metagenomes via a dereplication, aggregation and scoring strategy. Nature Microbiology 3, 836-843 (2018).

18 Sunagawa, S. et al. Structure and function of the global ocean microbiome. Science 348, 1261359 (2015).

19 Ainsworth, T. D. et al. The coral core microbiome identifies rare bacterial taxa as ubiquitous endosymbionts. The ISME Journal 9, 2261-2274 (2015).

20 Spoerner, M., Wichard, T., Bachhuber, T., Stratmann, J. \& Oertel, W. Growth and thallus morphogenesis of Ulva mutabilis (Chlorophyta) depends on a combination of two bacterial species excreting regulatory factors. Journal of Phycology 48, 14331447 (2012).

21 Lee, K. S. et al. An automated Raman-based platform for the sorting of live cells by functional properties. Nature Microbiology In Press (2019). 
61122 Lagier, J.-C. et al. Culturing the human microbiota and culturomics. Nature Reviews

23 Garcias-Bonet, N., Arrieta, J. M., Duarte, C. M. \& Marbà, N. Nitrogen-fixing bacteria in Mediterranean seagrass (Posidonia oceanica) roots. Aquatic Botany 131, 57-60 (2016).

24 Welsh, R. M. et al. Bacterial predation in a marine host-associated microbiome. The ISME Journal 10, 1540-1544 (2016).

25 Certner, R. H. \& Vollmer, S. V. Inhibiting bacterial quorum sensing arrests coral disease development and disease-associated microbes. Environmental microbiology 20, 645-657 (2018).

26 Mejia, A. Y. et al. Assessing the ecological status of seagrasses using morphology, biochemical descriptors and microbial community analyses. A study in Halophila Stipulacea (Forsk.) Aschers meadows in the northern Red Sea. Ecological Indicators 60, 1150-1163 (2016).

27 Glasl, B. et al. Microbiome variation in corals with distinct depth distribution ranges across a shallow-mesophotic gradient (15-85 m). Coral Reefs 36, 447-452 (2017).

28 Lachnit, T., Meske, D., Wahl, M., Harder, T. \& Schmitz, R. Epibacterial community patterns on marine macroalgae are host-specific but temporally variable. Environmental Microbiology 13, 655-665, doi:10.1111/j.1462-2920.2010.02371.x (2011).

29 Kimes, N. E. et al. The Montastraea faveolata microbiome: ecological and temporal influences on a Caribbean reef-building coral in decline. Environmental microbiology 15, 2082-2094 (2013).

30 Simister, R., Taylor, M. W., Rogers, K. M., Schupp, P. J. \& Deines, P. Temporal molecular and isotopic analysis of active bacterial communities in two New Zealand sponges. FEMS Microbiology Ecology 85, 195-205 (2013).

31 Lema, K. A., Bourne, D. G. \& Willis, B. L. Onset and establishment of diazotrophs and other bacterial associates in the early life history stages of the coral Acropora millepora. Molecular ecology 23, 4682-4695 (2014).

32 Gantt, S. E., López-Legentil, S. \& Erwin, P. M. Stable microbial communities in the sponge Crambe crambe from inside and outside a polluted Mediterranean harbor. FEMS Microbiol. Let. 364, fnx105 (2017).

33 Cúcio, C., Engelen, A. H., Costa, R. \& Muyzer, G. Rhizosphere microbiomes of European seagrasses are selected by the plant, but are not species specific. Frontiers in Microbiology 7, 440 (2016).

34 van de Water, J. A. J. M. et al. Seasonal Stability in the Microbiomes of Temperate Gorgonians and the Red Coral Corallium rubrum Across the Mediterranean Sea. Microb. Ecol. 75, 1-15, doi:10.1007/s00248-017-1006-y (2017).

35 Douglas, A. E. Symbiosis as a general principle in eukaryotic evolution. Cold Spring Harbor Perspectives in Biology 6, a016113 (2014).

36 Kelly, L. W. et al. Local genomic adaptation of coral reef-associated microbiomes to gradients of natural variability and anthropogenic stressors. Proceedings of the National Academy of Sciences 111, 10227-10232 (2014).

37 Janzen, D. H. When is it coevolution? Evolution 34, 611-612 (1980).

38 Thomas, T. et al. Diversity, structure and convergent evolution of the global sponge microbiome. Nature Communications 7, 11870 (2016).

39 Easson, C. G. \& Thacker, R. W. Phylogenetic signal in the community structure of host-specific microbiomes of tropical marine sponges. Frontiers in Microbiology $\mathbf{5}$, 532 (2014).

40 Borges, R. M. Co-niche construction between hosts and symbionts: ideas and evidence. Journal of Genetics 96, 483-489 (2017).

41 Koskella, B., Hall, L. J. \& Metcalf, C. J. E. The microbiome beyond the horizon of ecological and evolutionary theory. Nature ecology \& evolution 1, 1606 (2017). 
664

665

666

667

668

669

670

671

672

673

674

675

676

677

678

679

680

681

682

683

684

685

686

687

688

689

690

691

692

693

694

695

696

697

698

699

700

701

702

703

704

705

706

707

708

709

710

711

712

713

714

715

716

717

718

42 Brooks, A. W., Kohl, K. D., Brucker, R. M., van Opstal, E. J. \& Bordenstein, S. R. Phylosymbiosis: relationships and functional effects of microbial communities across host evolutionary history. PLoS biology 14, e2000225 (2016).

43 Pollock, F. J. et al. Coral-associated bacteria demonstrate phylosymbiosis and cophylogeny. Nature Communications 9, 4921 (2018).

44 Ramsby, B. D., Hoogenboom, M. O., Whalan, S. \& Webster, N. S. Elevated seawater temperature disrupts the microbiome of an ecologically important bioeroding sponge. Molecular Ecology 27, 2124-2137 (2018).

45 Brothers, C. J. et al. Ocean warming alters predicted microbiome functionality in a common sea urchin. Proceedings of the Royal Society B: Biological Sciences 285, 20180340 (2018).

46 Glasl, B., Smith, C. E., Bourne, D. G. \& Webster, N. S. Disentangling the effect of host-genotype and environment on the microbiome of the coral Acropora tenuis. PeerJ 7, e6377 (2019).

47 Thompson, J. N. The geographic mosaic of coevolution. (University of Chicago Press, 2005).

48 Singh, R. P. \& Reddy, C. Unraveling the functions of the macroalgal microbiome. Frontiers in microbiology 6, 1488 (2016).

49 Garren, M. et al. A bacterial pathogen uses dimethylsulfoniopropionate as a cue to target heat-stressed corals. The ISME journal 8, 999 (2014).

50 Kubanek, J. et al. Seaweed resistance to microbial attack: a targeted chemical defense against marine fungi. Proceedings of the National Academy of Sciences 100, 6916-6921 (2003).

51 Burke, C., Steinberg, P., Rusch, D., Kjelleberg, S. \& Thomas, T. Bacterial community assembly based on functional genes rather than species. Proceedings of the National Academy of Sciences 108, 14288-14293, doi:10.1073/pnas.1101591108 (2011).

52 Bulgarelli, D. et al. Revealing structure and assembly cues for Arabidopsis rootinhabiting bacterial microbiota. Nature 488, 91-95 (2012).

53 Rosado, P. M. et al. Marine probiotics: increasing coral resistance to bleaching through microbiome manipulation. The ISME Journal 13, 921-936 (2018).

54 Sodré, V. et al. Physiological aspects of mangrove (Laguncularia racemosa) grown in microcosms with oil-degrading bacteria and oil contaminated sediment.

Environmental Pollution 172, 243-249 (2013).

55 Egan, S. et al. The seaweed holobiont: understanding seaweed-bacteria interactions. FEMS Microbiology Reviews 37, 462-476 (2013).

56 Webster, N. S. \& Thomas, T. The Sponge Hologenome. mBio 7, e00135-00116, doi:10.1128/mBio.00135-16 (2016).

57 Meyle, J. \& Chapple, I. Molecular aspects of the pathogenesis of periodontitis. Periodontology 2000 69, 7-17 (2015).

58 Parekh, P. J., Balart, L. A. \& Johnson, D. A. The influence of the gut microbiome on obesity, metabolic syndrome and gastrointestinal disease. Clinical and translational gastroenterology 6, e91 (2015).

59 Egan, S. \& Gardiner, M. Microbial dysbiosis: Rethinking disease in marine ecosystems. Frontiers in Microbiology 7, 991 (2016).

60 Mera, H. \& Bourne, D. G. Disentangling causation: complex roles of coral-associated microorganisms in disease. Environmental microbiology 20, 431-449 (2018).

61 Campbell, A. H., Harder, T., Nielsen, S., Kjelleberg, S. \& Steinberg, P. D. Climate change and disease: bleaching in a chemically-defended seaweed. Global Change Biology 17, 2958-2970 (2011).

62 Peixoto, R. S., Rosado, P. M., Leite, D. C., Rosado, A. S. \& Bourne, D. G. Beneficial Microorganisms for Corals (BMC): Proposed Mechanisms for Coral Health and Resilience. Frontiers in Microbiology 8, 341, doi:10.3389/fmicb.2017.00341 (2017).

Sheaves, M. Consequences of ecological connectivity: the coastal ecosystem mosaic. Marine Ecology Progress Series 391, 107-115 (2009). 
$71964 \quad$ Bates, A. E. et al. Biologists ignore ocean weather at their peril. Nature 560, 299-301

65 Simon, J.-C., Marchesi, J. R., Mougel, C. \& Selosse, M.-A. Host-microbiota interactions: from holobiont theory to analysis. Microbiome 7, 5 (2019).

66 Cuellar-Gempeler, C. \& Leibold, M. A. Key colonist pools and habitat filters mediate the composition of fiddler crab-associated bacterial communities. Ecology 100, e02628 (2019).

67 Booth, J. M., Fusi, M., Marasco, R., Mbobo, T. \& Daffonchio, D. Fiddler crab bioturbation determines consistent changes in bacterial communities across contrasting environmental conditions. Scientific Reports 9, 3749 (2019).

68 Qiu, Z. et al. Future climate change is predicted to affect the microbiome and condition of habitat-forming kelp. Proceedings of the Royal Society B-Biological Sciences 286, 20181887 (2019).

69 Douglass, J. G., France, K. E., Richardson, J. P. \& Duffy, J. E. Seasonal and interannual change in a Chesapeake Bay eelgrass community: insights into biotic and abiotic control of community structure. Limnology and Oceanography 55, 14991520 (2010).

70 Casey, J. M., Connolly, S. R. \& Ainsworth, T. D. Coral transplantation triggers shift in microbiome and promotion of coral disease associated potential pathogens. Scientific reports 5, srep11903 (2015).

71 Campbell, A. H., Marzinelli, E. M., Gelber, J. \& Steinberg, P. D. Spatial variability of microbial assemblages associated with a dominant habitat-forming seaweed.

Frontiers in microbiology 6, 230 (2015).

72 Lafferty, K. D. \& Holt, R. D. How should environmental stress affect the population dynamics of disease? Ecology Letters 6, 654-664 (2003).

73 Huey, R. B. \& Stevenson, R. D. Integrating Thermal Physiology and Ecology of Ectotherms: A Discussion of Approaches. American Zoologist 19, 357-366, doi:10.1093/icb/19.1.357 (1979).

74 Vega Thurber, R. et al. Metagenomic analysis of stressed coral holobionts. Environmental Microbiology 11, 2148-2163, doi:10.1111/j.1462-2920.2009.01935.x (2009).

75 Fan, L., Liu, M., Simister, R., Webster, N. S. \& Thomas, T. Marine microbial symbiosis heats up: the phylogenetic and functional response of a sponge holobiont to thermal stress. The ISME Journal 7, 991-1002 (2013).

76 Lokmer, A. \& Mathias Wegner, K. Hemolymph microbiome of Pacific oysters in response to temperature, temperature stress and infection. The ISME Journal 9, 670682, doi:10.1038/ismej.2014.160 (2015).

77 Case, R. J. et al. Temperature induced bacterial virulence and bleaching disease in a chemically defended marine macroalga. Environmental Microbiology 13, 529-537, doi:10.1111/j.1462-2920.2010.02356.x (2011).

78 Greff, S. et al. The interaction between the proliferating macroalga Asparagopsis taxiformis and the coral Astroides calycularis induces changes in microbiome and metabolomic fingerprints. Scientific Reports 7, 42625 (2017).

79 Brown, A. L., Lipp, E. K. \& Osenberg, C. W. Algae dictate multiple stressor effects on coral microbiomes. Coral Reefs 38, 229-240, doi:10.1007/s00338-019-01769-w (2019).

80 van der Heide, T. et al. A three-stage symbiosis forms the foundation of seagrass ecosystems. Science 336, 1432-1434, doi:10.1126/science.1219973 (2012).

81 Roth-Schulze, A. J. et al. The effects of warming and ocean acidification on growth, photosynthesis, and bacterial communities for the marine invasive macroalga Caulerpa taxifolia. Limnology and Oceanography 63, 459-471 (2018).

82 Banerjee, S., Schlaeppi, K. \& Heijden, M. G. Keystone taxa as drivers of microbiome structure and functioning. Nature Reviews Microbiology 16, 567-576 (2018). 
77283 Jaspers, C. et al. Resolving structure and function of metaorganisms through a

84 Bang, C. et al. Metaorganisms in extreme environments: do microbes play a role in organismal adaptation? Zoology 127, 1-19 (2018).

85 Tyagi, M., da Fonseca, M. M. R. \& de Carvalho, C. C. Bioaugmentation and biostimulation strategies to improve the effectiveness of bioremediation processes. Biodegradation 22, 231-241 (2011).

86 Nikolopoulou, M. \& Kalogerakis, N. Biostimulation strategies for fresh and chronically polluted marine environments with petroleum hydrocarbons. Journal of Chemical Technology and Biotechnology 84, 802-807 (2009).

87 Poi, G., Aburto-Medina, A., Mok, P. C., Ball, A. S. \& Shahsavari, E. Large scale bioaugmentation of soil contaminated with petroleum hydrocarbons using a mixed microbial consortium. Ecological Engineering 102, 64-71 (2017).

88 Yu, K., Wong, A., Yau, K., Wong, Y. \& Tam, N. Natural attenuation, biostimulation and bioaugmentation on biodegradation of polycyclic aromatic hydrocarbons (PAHs) in mangrove sediments. Marine Pollution Bulletin 51, 1071-1077 (2005).

89 Finkel, O. M., Castrillo, G., Herrera Paredes, S., Salas González, I. \& Dangl, J. L. Understanding and exploiting plant beneficial microbes. Current Opinion in Plant Biology 38, 155-163, doi:https://doi.org/10.1016/j.pbi.2017.04.018 (2017).

90 Slattery, J., MacFabe, D. F. \& Frye, R. E. The significance of the enteric microbiome on the development of childhood disease: A review of prebiotic and probiotic therapies in disorders of childhood. Clinical Medicine Insights. Pediatrics 10, 91 (2016).

91 Carnevali, O., Maradonna, F. \& Gioacchini, G. Integrated control of fish metabolism, wellbeing and reproduction: The role of probiotic. Aquaculture 472, 144-155 (2017).

92 Oppen, M. J. et al. Shifting paradigms in restoration of the world's coral reefs. Global Change Biology 23, 3437-3448 (2017).

93 Stewart, J. R. et al. The coastal environment and human health: microbial indicators, pathogens, sentinels and reservoirs. Environmental Health 7, S3 (2008).

94 Takemura, A. F., Chien, D. M. \& Polz, M. F. Associations and dynamics of Vibrionaceae in the environment, from the genus to the population level. Frontiers in Microbiology 5, 38 (2014).

95 Ralston, E. P., Kite-Powell, H. \& Beet, A. An estimate of the cost of acute food and water borne health effects from marine pathogens and toxins in the United States. Journal of Water and Health 9, 680-694 (2011).

96 Lamb, J. B. et al. Seagrass ecosystems reduce exposure to bacterial pathogens of humans, fishes, and invertebrates. Science 355, 731-733 (2017).

97 Halpern, B. S. et al. Spatial and temporal changes in cumulative human impacts on the world's ocean. Nature Communications 6, 7615 (2015).

98 Labbate, M., Seymour, J. R., Lauro, F. \& Brown, M. V. Anthropogenic impacts on the microbial ecology and function of aquatic environments. Frontiers in Microbiology 7 , 1044 (2016).

99 Glasl, B., Webster, N. S. \& Bourne, D. G. Microbial indicators as a diagnostic tool for assessing water quality and climate stress in coral reef ecosystems. Marine Biology 164, 91 (2017).

100 Gillings, M. R. et al. Using the class 1 integron-integrase gene as a proxy for anthropogenic pollution. The ISME Journal 9, 1269-1279 (2015).

101 McLellan, S. L. \& Eren, A. M. Discovering new indicators of fecal pollution. Trends in Microbiology 22, 697-706 (2014).

102 Fisher, J. C. et al. Comparison of sewage and animal fecal microbiomes by using oligotyping reveals potential human fecal indicators in multiple taxonomic groups. Applied Environmental Microbiology 81, 7023-7033 (2015).

103 Fisher, J. C., Levican, A., Figueras, M. J. \& McLellan, S. L. Population dynamics and ecology of Arcobacter in sewage. Frontiers in Microbiology 5, 525 (2014). 
827104 Knights, D. et al. Bayesian community-wide culture-independent microbial source

105 Roguet, A., Eren, A. M., Newton, R. J. \& McLellan, S. L. Fecal source identification using random forest. Microbiome 6, 185 (2018).

106 Moitinho-Silva, L. et al. The sponge microbiome project. GigaScience 6, 1-7 (2017).

107 Tripathi, A. et al. Are microbiome studies ready for hypothesis-driven research? Current Opinion in Microbiology 44, 61-69 (2018).

108 Sutherland, W. J. et al. Identification of 100 fundamental ecological questions. Journal of ecology 101, 58-67 (2013).

109 Cook, C. N., Inayatullah, S., Burgman, M. A., Sutherland, W. J. \& Wintle, B. A. Strategic foresight: how planning for the unpredictable can improve environmental decision-making. Trends in Ecology \& Evolution 29, 531-541 (2014).

110 Hemming, V., Burgman, M. A., Hanea, A. M., McBride, M. F. \& Wintle, B. C. A practical guide to structured expert elicitation using the IDEA protocol. Methods in Ecology and Evolution 9, 169-180 (2018).

111 Martin, T. G. et al. Eliciting expert knowledge in conservation science. Conservation Biology 26, 29-38 (2012).

112 Feehery, G. R. et al. A method for selectively enriching microbial DNA from contaminating vertebrate host DNA. PloS one 8, e76096 (2013).

113 Eren, A. M. et al. Oligotyping: differentiating between closely related microbial taxa using 16S rRNA gene data. Methods in Ecology and Evolution 4, 1111-1119 (2013).

114 Callahan, B. J., McMurdie, P. J. \& Holmes, S. P. Exact sequence variants should replace operational taxonomic units in marker-gene data analysis. The ISME Journal 11, 2639-2643 (2017).

115 Edgar, R. C. Updating the $97 \%$ identity threshold for 16 S ribosomal RNA OTUs. Bioinformatics 34, 2371-2375, doi:10.1093/bioinformatics/bty113 (2018).

116 Pogoreutz, C. et al. Nitrogen fixation aligns with nifH abundance and expression in two coral trophic functional groups. Frontiers in Microbiology 8, 1187 (2017).

117 Cardini, U. et al. Microbial dinitrogen fixation in coral holobionts exposed to thermal stress and bleaching. Environmental microbiology 18, 2620-2633 (2016).

118 Glasl, B., Herndl, G. J. \& Frade, P. R. The microbiome of coral surface mucus has a key role in mediating holobiont health and survival upon disturbance. The ISME Journal 10, 2280-2292 (2016).

119 Do Carmo, F. L. et al. Bacterial structure and characterization of plant growth promoting and oil degrading bacteria from the rhizospheres of mangrove plants. The Journal of Microbiology 49, 535 (2011).

120 Bourlat, S. J. et al. Genomics in marine monitoring: new opportunities for assessing marine health status. Marine Pollution Bulletin 74, 19-31 (2013). 
867 Correspondence and requests for materials should be addressed to S.T.T.

868

869 Acknowledgements:

870 We thank Deakin University's School of Life and Environmental Sciences and the Centre for

871 Integrative Ecology for funding the workshop (New Activities Scheme 2017). BL's

872 attendance at the workshop was funded by the New Zealand Ministry of Business, Innovation

873 \& Employment (MBIE) Smart Ideas project UOWX1602. LM's attendance was supported

874 by an Australian Research Council Discovery Project (DP160103811). AC's attendance

875 was funded by the Centre for Marine Bio-Innovation at UNSW Australia. AHE was

876 supported by fellowship SFRH/BPD/107878/2015 from the Portuguese Science

877 Foundation (FCT). DD was supported by King Abdullah University of Science and

878 Technology (baseline research funds). STT was supported by Deakin University's SEBE

879 Postdoctoral Industry Fellowship, the Mary Collins Trust and the Alfred Deakin

880 Postdoctoral Research Fellowship. We thank Jennifer Martiny, Steven Robbins, Gene

881 Tyson, Claudia Weihe and Katrine Whiteson for their input in the horizon scanning

882 exercise. We thank Leena Koop for producing the conceptual designs.

883

884

885 Author Contributions:

886 S.T.-T. conceived the idea; S.T.-T., C.S. and P.I.M. developed and led the workshop; S.T.-T.,

887 C.S., P.I.M., M.J.H., A.H.C., B.L., V.H.-M., J.S., L.M., T.A. and K.L.H. attended the 888 workshop that structured the themes of the manuscript; S.T.-T., C.S., P.I.M., M.J.H., A.H.C.,

889 B.L., V.H.-M., J.S., L.M., T.A., K.L.H., A.F., D.D., S.E., A.H.E., M.F., T.T., L.V., A.H.-A.,

890 H.M.G., E.M.M. and P.D.S. contributed to the questions and wrote the manuscript; A.F.,

891 V.H.-M., S.T.-T. and L.H. contributed to the literature search. 
893 The authors have no competing interests.

894 
896

897 Fig. 1: Determining microbiome contribution to coastal ecosystem health. a. This paper on 898 coastal marine microbiomes highlights six key holobionts that form the foundation of coastal 899 ecosystems: corals, macroalgae, seagrasses, mangroves (sponges and saltmarshes not shown 900 here). b. The core microbiome concept allows the identification of both persistent microbial 901 phylotypes, and c. core functional roles played by microbes within holobionts, seawater and 902 the sediment. Different microbes can constitute persistent microbiomes across varying 903 spatial, temporal, organism and ecosystem scales (intersection in Venn diagram, b).

904 However, these ubiquitous microbial communities are likely to present functional redundancy 905 across coastal environments (similar relative abundance in functional genes, c), playing

906 crucial roles in the functioning and health of coastal marine ecosystems. OTU = operational

907 taxonomic unit, KEGG = Kyoto Encyclopedia of Genes and Genomes. Photo credits: coral

908 reef: Alexander J. Fordyce; mangrove: Michael Bradley; seagrass and macroalgae:

909 Pommeyrol Vincent and Ethan Daniels, respectively / Shutterstock.

910

911 Fig. 2: Conceptual design of the potential relationships between coastal marine microbiomes

912 and humans. (1) Coastal pollution, including stormwater and sewage effluence, introduce

913 potentially pathogenic microbiota to coastal marine ecosystems. (2) Endemic marine

914 pathogens, including Vibrio and toxic cyanobacteria, persist in coastal marine ecosystems. (3)

915 Coastal aquaculture species can become contaminated by endemic and introduced pathogens,

916 which can both (i) cause mortality, e.g. oysters, and (ii) have health implications for human

917 consumers. (4) Endemic pathogens, e.g. Vibrio, can cause holobiont disease. (5) According to

918 Lamb et al. ${ }^{96}$, coastal macrophytes may act as natural pathogen filters, buffering the impact

919 of pathogens for humans and coastal marine ecosystems. Climate change and $\mathrm{CO}_{2}$-mediated 
920 pollution also represent major impacts that humans have on coastal microbiomes. These

921 impacts, for example, may result in increased occurrence and virulence of pathogens like

922 Vibrio via the warming of sea surface temperatures (2), and increased coastal pollution due to

923 the greater frequency of storm events (1). The industry and global warming symbols are

924 courtesy of the Integration and Application Network, University of Maryland Center for

925 Environmental Science (ian.umces.edu/symbols/).

926

927 Fig. 3: A conceptual diagram depicting several of the major research themes in coastal 928 marine microbiome research. The diagram highlights several interactions that occur at 929 multiple spatial levels. a. Using the macroalgae Ulva sp. as an example, the inset highlights

930 six host-microbiome interactions and associations in relation to the 'baseline' holobiont

931 (center host + microbiome). b, c. More broadly, the holobiont-scale of interactions and

932 associations can also apply to large-scale or ecosystem-level scenarios, whereby the

933 holobiont interacts with environmental microbiomes (e.g. sediments/substrates, seawater) and 934 neighboring inter- and intra-species holobionts, while also being influenced by environmental 935 or climatic conditions (not depicted here).

936 
938

939

\section{Box 1: Key Definitions}

Dysbiosis: An imbalance or disruption of the normally beneficial symbiotic relationship between the host and its associated microbiota. A dysbiotic microbiota may result in poor host health and/or reduced capacity for resistance to environmental perturbation.

Holobiont: An ecological unit formed by a host and its associated microbiome(s).

Horizon scanning: A technique used to systematically identify the gaps, challenges and opportunities in a field with the aim to outline future priorities and is often employed by eliciting the perspectives of experts in the field.

Metagenomics: The study of microbial community structure, function and interactions through the sequencing and analysis of genetic material directly extracted from the environment.

Metatranscriptomics: The study of the expressed genes in an environment or holobiont.

Microbiome: The sum of the microbial consortia (and their genetic material) in an environment. The microbiome typically includes a diversity of prokaryotes (bacteria and archaea), eukaryotes (fungi and protozoa) and viruses.

Operational taxonomic unit (OTU): Marker genes from multiple individuals that were clustered/grouped on the basis of sequence similarity to represent a taxonomic group.

Phenotype: The observable characteristics of an organism, influenced by genetics and the environment.

Phylogenetic marker genes: A genetic marker whose sequence is used to delineate taxonomic and evolutionary relationships. Examples are the 16S rRNA gene in prokaryotes and 18S rRNA gene and ITS (internal transcribed spacer regions) in eukaryotes.

Quorum sensing: The ability to regulate gene expression in response to changes in cellpopulation density through the production and detection of specific chemical signal molecules (autoinducers) within or among populations. 


\section{Box 2: Literature Search and Horizon Scan Methodology}

Approach: First, we surveyed the literature to identify the breadth of research to-date and evaluate the state of understanding in the field of coastal, estuarine, and marine microbiome research. A detailed description of the literature search, including the script that was used, can be found in Supplementary Methods. In brief, the SCOPUS database was used for searching, with keywords including: seagrass, mangrove, saltmarsh, macroalgae, coral, sponge, seaweed, seawater and sediment. This resulted in 671 publications, after irrelevant publications were excluded. We then manually scanned these publications to extract information on methods, host type, and GPS location of sampling site. The content of these papers was then used quantitatively and qualitatively in this paper to report on the state of the science.

Second, we used a modified horizon scanning method ${ }^{108}$ to identify key questions in coastal marine microbiome research. This approach uses expert solicitation to provide strategic foresight into the key research gaps for future research; an approach that has been historically underutilized in environmental science ${ }^{109}$, yet can provide a powerful approach to prioritizing a research agenda when appropriately structured ${ }^{110,111}$. Briefly (more details provided in Supplementary Data 1), the approach involved asking experts in the field of microbiome research to freely contribute what they considered to be the most pending questions (10 maximum) in coastal and estuarine microbiome science - i.e. what are the major research gaps and where do the challenges lie? Experts were initially selected from within the lead and last author's research networks and based on who the lead/last author perceived to be at the forefront of the research field through the aforementioned literature search. To this end, the selection of authors could be considered haphazard.

A total of 34 experts were approached by email, spanning 12 institutions from Australia, Europe, Saudi Arabia and the United States (US). Of the invited experts, 28 experts (84\%) contributed responses (after two email reminders) and submitted 108 questions collectively. A workshop was held at Deakin University (Melbourne, Australia) on 6-7 July 2017 to refine the questions based on the voting process as described in Sutherland et al. ${ }^{108}$. The questions were grouped into major research themes and are presented herein to discuss the research gaps in the context of the questions that underpinned them. Therefore, the themes presented below are not in a particular order of importance; they are arranged in a way that starts with 
microbiome-centric concepts, to relationships between microbiome and host, and lastly to broader interactions within and across microbiomes, the holobiont and the environment (Box Diagram).

1005

[suggested Box Diagram placement. Caption below]

1007

1008

Box Diagram: Conceptual design depicting the seven themes resulting from the horizon

1009

1010

1011

1012 scanning exercise.

Limitations: Horizon scanning approaches have limitations, which, most importantly, include the risk of questions being inherently biased by the interests of the researchers ${ }^{108}$. To limit this bias, we made an effort to solicit questions from researchers across a wide range of sub-fields, including plankton, sediment/substrate, seagrass, seaweed, coral, sponge and mangrove microbiomes. Additionally, while the solicited scientists work internationally, we were aware of potential biogeographic biases that could influence the questions. Therefore, in the original request for questions, we asked the scientists to keep their questions global in order to avoid national- or regional-specific topics.

1018

1019 


\section{Box 3: Methodological challenges}

1. Molecular Approaches

DNA and RNA sequencing has been increasingly used as the preferred technique in coastal marine microbiome studies (Supplementary Fig. 1), yet several challenges have the potential to limit the production of reliable datasets. Such molecular challenges are broadly found in microbiome research, so we outline two current challenges in coastal marine microbiome research and suggest promising techniques that could help overcome these issues:

\section{Challenges}

- Host genome contamination in (meta)genomic studies on host-associated microbiomes

Bioinformatic challenges

- The level of taxonomic resolution needed in order to address questions on microbial composition and function

- $\quad$ The arbitrarily defined $97 \%$ sequence similarity designation of operational taxonomic units (OTUs)
- Pre-and post-sequencing removal of contaminating host cells and DNA sequences via

- Physical removal of host tissue, e.g. centrifugation, Percoll separation

- In-silico removal of well-curated host DNA sequences post-sequencing

- Removal of methylated eukaryotic host DNA*112

○ Host-specific blocking primers

- Employ amplicon sequencing approaches using universal primers as a first step (e.g. optimal gene segments V3 and V4), with added approaches, such as meta-'omics', for a more comprehensive understanding on microbial dynamics and functional roles.

- Use the most up-to-date and statistically valid methods for inferring the highest taxonomic resolution, e.g. ${ }^{113-115}$. Benefits include

- Higher resolution profiles of microbial communities in a unit

- Directly comparable between datasets

- Genotype discrimination could also be improved by longer sequences

\section{Manipulative Experiments}

Laboratory manipulative experiments are key to addressing hypotheses in many of the research themes addressed here. Yet, while field microbiome experiments are essential to answer questions under natural, real-world conditions, field manipulations of host-microbiome interactions in coastal marine ecosystems are rare ${ }^{116,117}$. Challenges include the logistics of excluding prokaryotes in the environment e.g. sterilization or antibiotics, but, for holobionts that are easily transportable, could be overcome with antibiotic treatment in the laboratory before field deployment ${ }^{118}$. Such a combination of innovatively designed laboratory and field experiments likely hold the key to teasing out important microbiome and holobiont interactions. Experiments that exclude or add specific microbes, resources, or isotope tracers would be useful in understanding functions and fine-scale interactions (e.g. beneficial microbiota ${ }^{53}$ ), while the manipulation of environmental conditions could be used to simulate climate change, stress, or pollution scenarios (e.g. adding oil-degrading and plant growth promoting bacteria to oil spills in mangrove forests $\left.^{54,119}\right)$. Additionally, large simulator laboratories and in situ manipulative experiments could be a potential middle ground for testing hypotheses that have inherent field challenges ${ }^{44}$.

\section{Coastal Marine Microbiome Management}

We discussed in Themes 4 and 6 the possibilities of managing and manipulating microbes in coastal marine ecosystems to aid in pollution and eutrophication remediation, restoration, disease management and enhancing host health and growth. With the current momentum in this space, we predict that several challenges and questions will arise, such as, 'Can a managed or manipulated microbiome outlive its function, and if so, what impacts does this have on the microbiome, holobiont or ecosystem?', 'Is there a way to 'stop' a microbial function or remove a community once a particular job has been done?', and 'How resilient would a managed or manipulated microbiome be to disturbances and how would they be monitored to know if a desirable or undesirable outcome is achieved?'. Lastly, we predict that one of the key challenges will be to incorporate applied microbiome research into local, regional and national policy and methodology. As Bourlat et al. ${ }^{120}$ also outlined, we suggest that stakeholders need to be identified and engaged at the state and national levels early on in the research.

*This will lead to the removal of any methylated DNA including that from protists and occasionally fungi, depending on the methylation rate. 
1020 


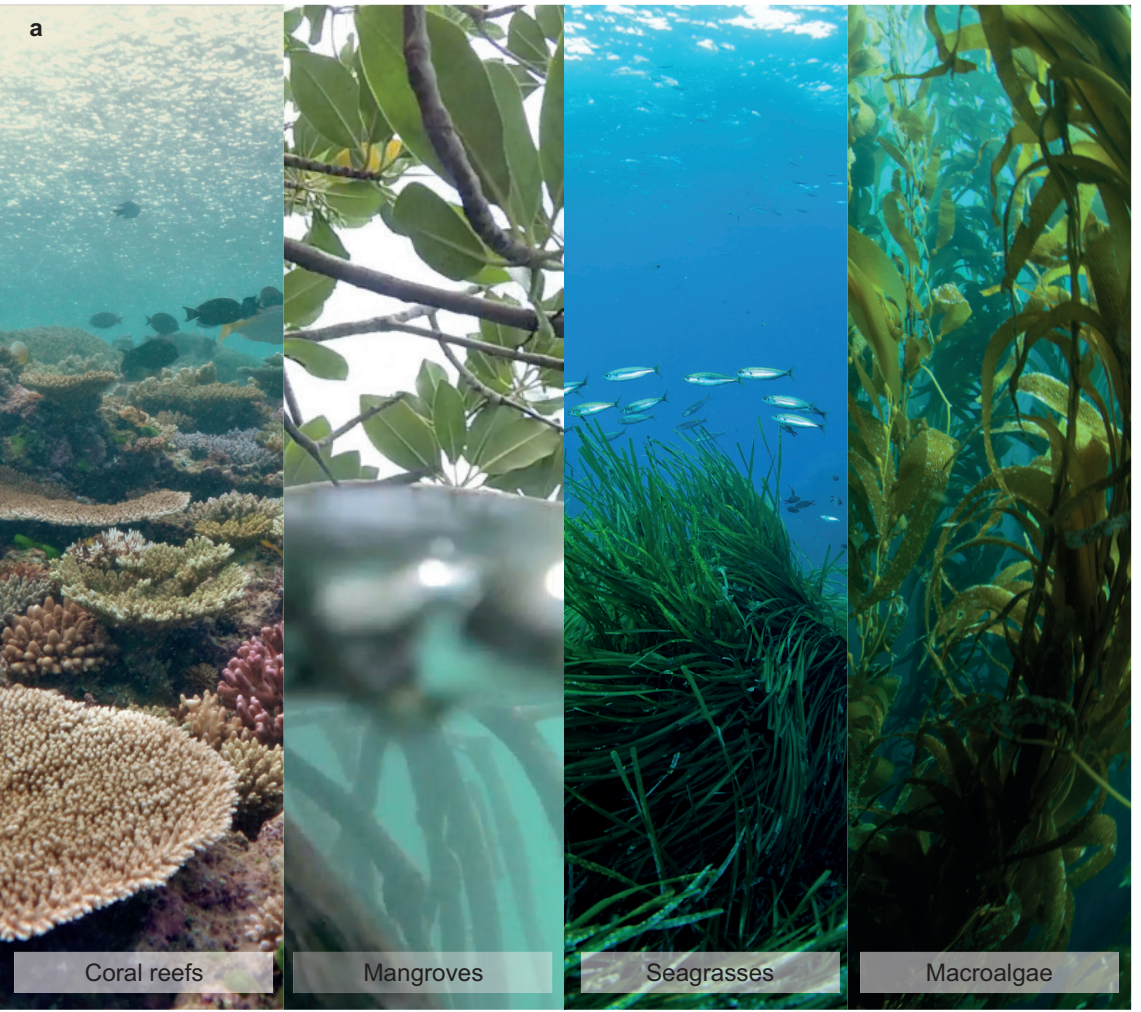

Core $/$ persistent microbiome $=3$ shared OTUs

c

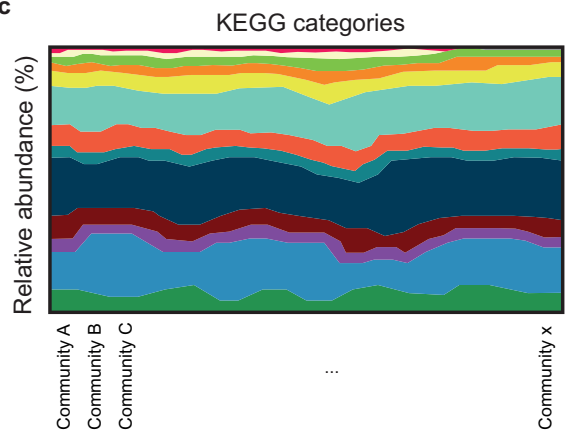

Core/persistent microbiome $=11$ functions 


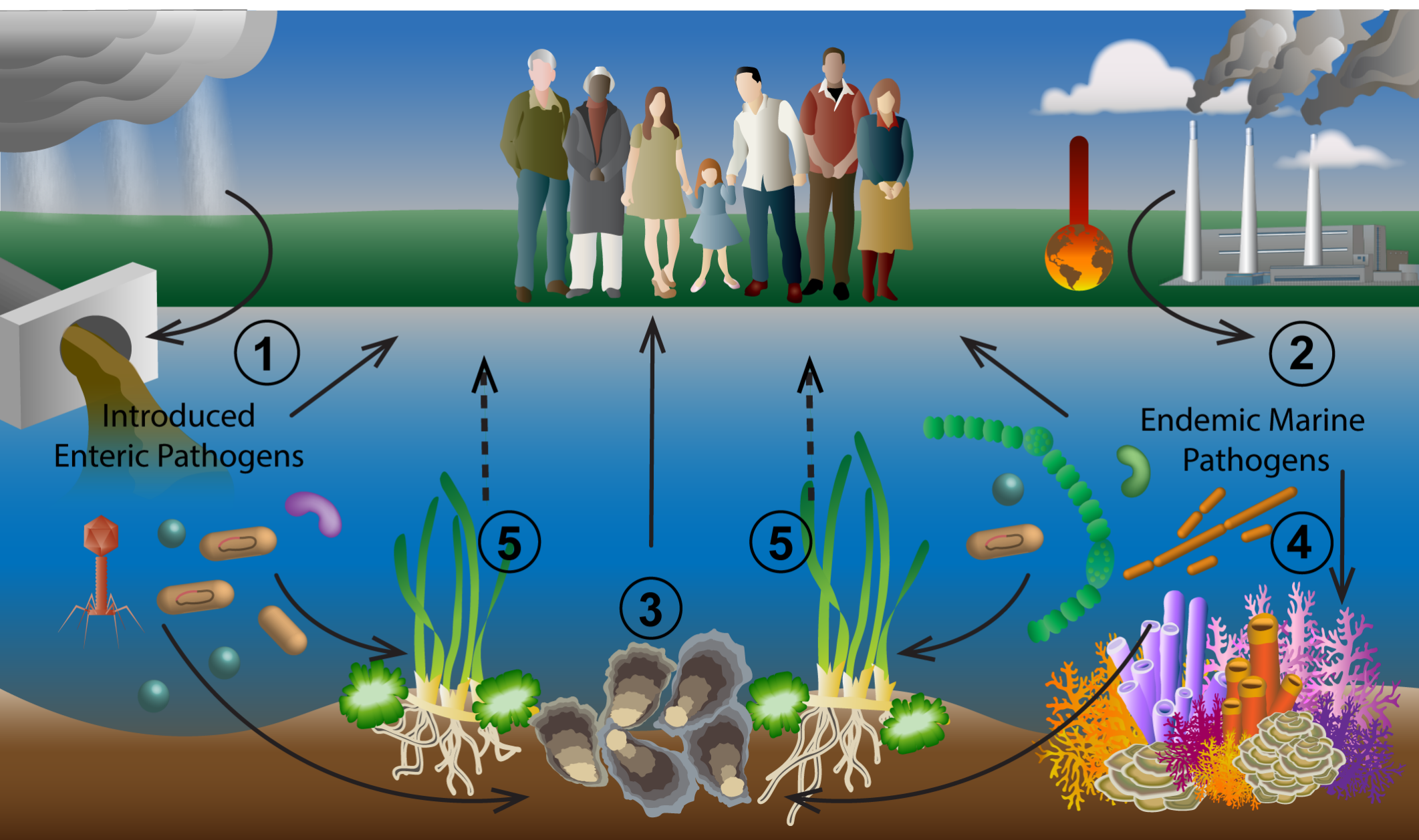




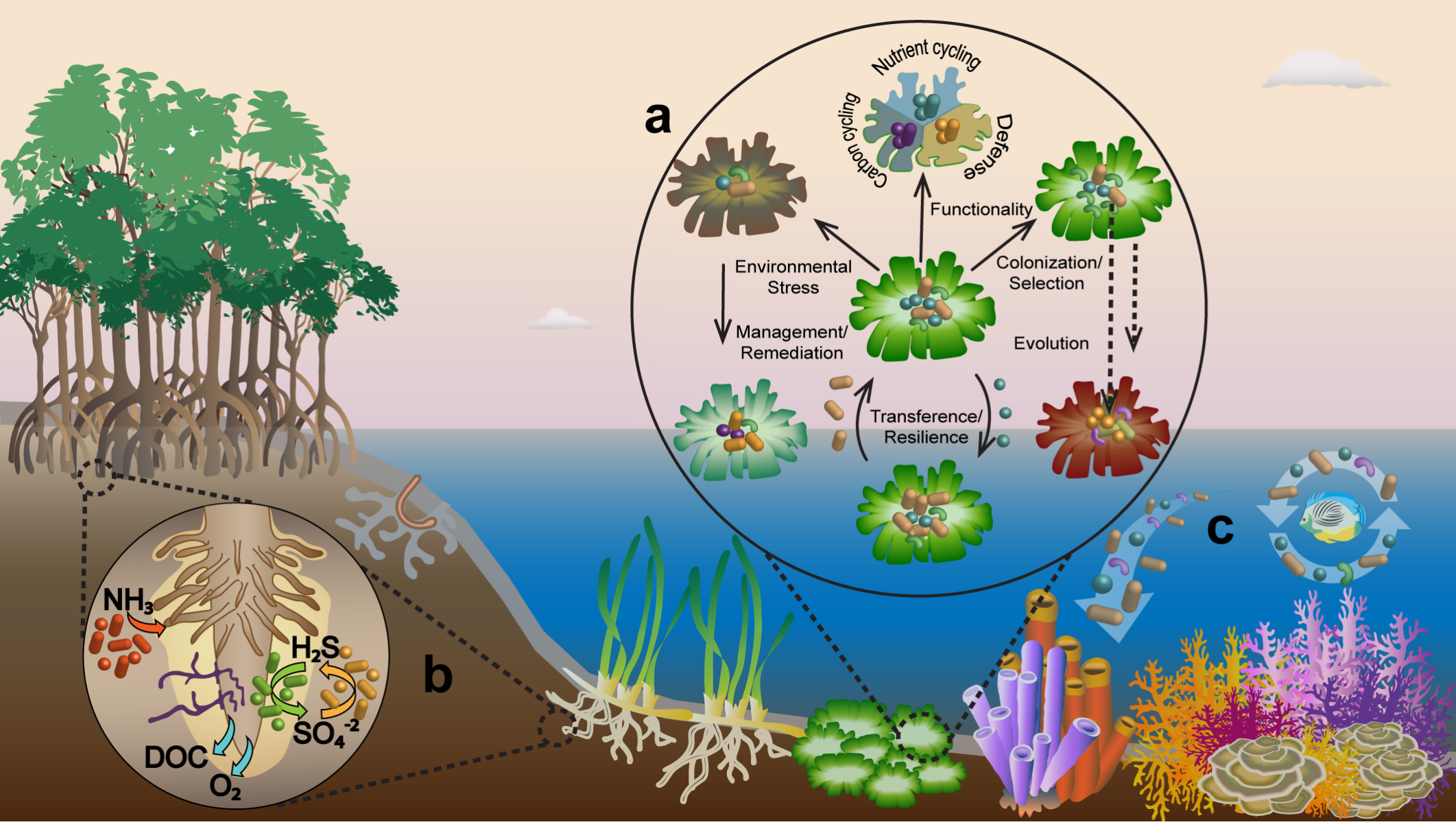




\section{Microbiome}

1: Defining

2: Scale

\section{Microbiome \& Host}

3: Assembly and disassembly

4: Resilience

\section{Holobiont \& Environment}

5: Tripartite interactions

6: Management

7: Human connection 